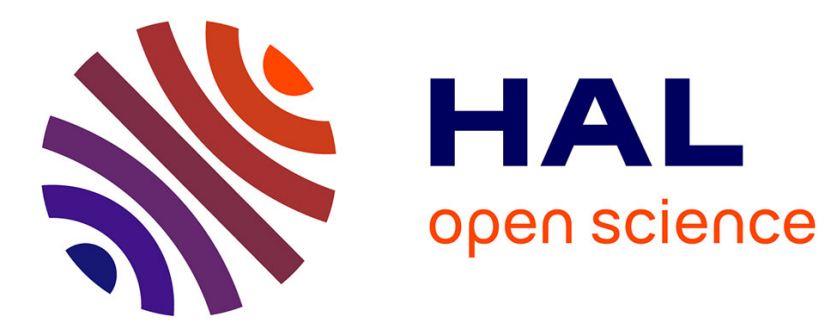

\title{
La vulgarisation excentrique pour tous: les leçons d'histoire naturelle de Pierre Boitard dans le Musée des familles
}

Azélie Fayolle

\section{- To cite this version:}

Azélie Fayolle. La vulgarisation excentrique pour tous : les leçons d'histoire naturelle de Pierre Boitard dans le Musée des familles. Littérature française et savoirs biologiques au XIXe siècle, De Gruyter, pp.213 - 232, 2019, 10.1515/9783110665833-015 . hal-03517572

\section{HAL Id: hal-03517572 \\ https://hal.science/hal-03517572}

Submitted on 7 Jan 2022

HAL is a multi-disciplinary open access archive for the deposit and dissemination of scientific research documents, whether they are published or not. The documents may come from teaching and research institutions in France or abroad, or from public or private research centers.
L'archive ouverte pluridisciplinaire HAL, est destinée au dépôt et à la diffusion de documents scientifiques de niveau recherche, publiés ou non, émanant des établissements d'enseignement et de recherche français ou étrangers, des laboratoires publics ou privés.

\section{(ㅇ)(1) $\$$}

Distributed under a Creative Commons Attribution - NonCommercial - NoDerivatives| 4.0 


\section{La vulgarisation excentrique pour tous : les leçons d'histoire naturelle de Pierre Boitard dans le Musée des familles}

Malgré une production vaste et protéiforme, le naturaliste et vulgarisateur Pierre Boitard n'apparaît pas au premier plan de l'espace médiatique du premier $\mathrm{XIX}^{\mathrm{e}}$ siècle, ni même de l'histoire des sciences naturelles. Ni académicien, ni théoricien de premier ordre, il publie des travaux scientifiques et de vulgarisation épars, parfois paradoxaux, et dont le ton peut se faire frondeur. C'est dans les collections de manuels et dans les pages des revues populaires que Boitard trouve sa place : si le prestige manque à ces publications, elles lui permettent par contre de rencontrer un public particulièrement large. En devenant une figure tutélaire du Musée des familles, Boitard met progressivement à profit la liberté de ton que rendent possible la modestie de ses lecteurs et la nécessité éditoriale de fournir des livraisons régulières. La revue du Musée des familles, revue destinée à un public familial, se trouve prise dans la double contrainte d'instruire en plaisant. Le ton badin de Boitard, volontiers farceur, trouve la possibilité de développer, à l'occasion de leçons plus ou moins romancées, des théories qui ne seront publiées en volume qu'après sa mort : les contraintes médiatiques du périodique sont alors transformées en autant d'opportunités par un naturaliste peu orthodoxe. Les contributions de Pierre Boitard au Musée des familles montrent un aspect méconnu d'un naturaliste apparemment secondaire : les premières esquisses de son roman scientifique, Paris avant les hommes (1861) se trouvent dans les publications du Musée des familles. Ce n'est cependant pas comme l'annonce d'un roman posthume qu'il faut lire ces leçons d'histoire naturelle : celles-ci doivent d'abord être considérées comme un témoignage des évolutions importantes des sciences naturelles de cette période. Il s'agit ainsi dans cet article de comprendre comment l'inscription de leçons scientifiques dans une revue populaire permet une autonomisation progressive des savoirs, et surtout du savant qui les présente. Placées à mi-chemin entre sciences et littérature, les leçons d'histoire naturelle de Pierre Boitard rappellent en effet les débats qui pouvaient agiter les naturalistes du premier $\mathrm{XIX}^{\mathrm{e}}$ siècle, notamment sur la généralisation des nomenclatures taxonomiques. Le format des leçons d'histoire naturelle, qui aurait pu manifester un certain académisme, se révèle propice à des détournements de la 
volonté affichée de transmission de savoirs, au point de conduire à une refondation du genre de la vulgarisation scientifique, jusqu'à un refus - provisoire - de la vulgarisation scientifique elle-même. Les marges des espaces médiatiques offrent alors à un naturaliste peu académique la possibilité de développer une science marginale, qui ridiculise son scientifique pour lui refuser tout prestige ou toute autorité. Cette vulgarisation scientifique paradoxale montre ainsi la prise en compte d'un nouveau format médiatique, et elle se joue des attentes de son public en ridiculisant son format comme son objet, la science comme les savants. Avant l'institutionnalisation des manuels scolaires ou pédagogiques, et avant l'ouverture de l'immense marché de la vulgarisation scientifique pendant la seconde moitié du XIX ${ }^{\mathrm{e}}$ siècle, les leçons d'histoire naturelle de Pierre Boitard donnent, dans le Musée des familles, un exemple rare de diffusion des savoirs comme de leur contestation.

\section{Le Musée des familles, une revue populaire du XIX ${ }^{\mathrm{e}}$ siècle}

Souvent éclipsé par Le Magasin pittoresque, son concurrent direct également riche en illustrations, le Musée des familles est lui-même oublié des études consacrées à la presse populaire du XIX ${ }^{\mathrm{e}}$ siècle. Fondé en 1833 par Émile de Girardin, le Musée des familles. Lectures du soir est une revue grand public inspirée du Penny Magazine anglais, illustrée de grandes planches dont une pleine page. La revue se veut d'emblée « plein[e] d'amusement [et] d'instruction », un " [j]ournal utile à tous, journal des hommes et des femmes, journal de Paris, journal étranger, journal de tous les hommes, de tous les pays, de tous les temps ; journal de la famille surtout, innocent et inoffensif, bien que malin et railleur. " ${ }^{1}$

Le Musée est livré par abonnement, à un rythme d'abord mensuel puis bimensuel, avec une pagination continue des numéros pour permettre la reliure en volumes. ${ }^{2}$ Émile de Girardin quitte rapidement la publication ; il est remplacé par le vulgarisateur S.-H. Berthoud, puis par Pitre-Chevalier de 1842 à sa mort en 1863. Le format des numéros évolue au fil des années de publication ; la revue présente des études d'histoire naturelle, des progrès techniques, de géographie,

1 Jules Janin : Les Magasins anglais. In : Musée des familles 1 (1833-1834), p. 3.

2 Des retirages ont cependant été constatés, pouvant amener une réorganisation (et donc, parfois, un changement de pagination) au sein d'une même année. 
des poèmes, chansons ou nouvelles, comme des visites de lieux emblématiques. Le ton des premières années peut se faire irrévérencieux, mais il reste plutôt édifiant et moralisateur, surtout à partir des années $1850 .^{3}$

AU: We have shortened therunning head. Please check and

Le Musée bénéficie pour son lancement d'une importante campagne de publicité placardée dans tout Paris. Grâce à ses nombreuses images et son bas prix, la revue connaît un succès foudroyant : les éditoriaux signalent 45000 abonnements en 1833, qui montent jusqu'à 52000 à la fin de 1834, mais baissent ensuite (42 000 en 1835, 29000 en 1837). Le Musée tire ensuite à 30000 en 1850, et il connaît son apogée au début des années 1860, sous la direction de Pitre-Chevalier. Des retirages sont proposés dès 1836. Le Musée des familles ne dépasse pas le XIXe siècle ; il cesse d'être publié en 1900, alors que son concurrent, Le Magasin pittoresque, poursuit ses publications jusqu'en 1938. Il est alors « universellement connu du public » : on le trouve " dans les bibliothèques bourgeoises, les bibliothèques publiques et même les bibliothèques de prisons où il est l'un des périodiques les plus consultés ». ${ }^{4}$ Par son ample diffusion et sa volonté constante de livrer " amusement » et " instruction ", ${ }^{5}$ le Musée des familles est ainsi une source de vulgarisation populaire à large diffusion. La revue se détourne des questions d'actualité, mais elle couvre le champ de la plupart des savoirs et elle accorde une place privilégiée aux sciences naturelles, dont des leçons sont données dès octobre 1833 par Nodier. Les années durant lesquelles Pierre Boitard publie ses leçons d'histoire naturelle et quelques récits dans le Musée des familles constituent un temps fort de la revue, qui joue alors un rôle de passeur culturel important pour les littératures contemporaines, françaises ou traduites ainsi que par ses leçons scientifiques sur des sujets variés. La collaboration de Pierre Boitard au Musée des familles, personnage lui-même polymathe, est caractéristique de l'hybridité générique des premières revues populaires du $\mathrm{XIX}^{\mathrm{e}}$ siècle.

3 La revue joue par ailleurs un rôle sous-estimé dans la diffusion de la vulgarisation scientifique dès la première moitié $\mathrm{du} \mathrm{XIX}^{\mathrm{e}}$ siècle, tout comme elle facilite un important transfert culturel : le magazine propose régulièrement une rubrique de littérature étrangère, dans laquelle on peut lire des textes de Washington Irving, Hoffmann ou Andersen, entre autres. Des articles sont d'ailleurs régulièrement traduits des journaux anglais. Parmi les plumes qui contribuent à la publication se trouvent les noms de Balzac, Hugo ou Dumas, Marceline Desbordes-Valmore, Lamartine, Delphine de Girardin et sa mère Sophie Gay, Nodier, Eugène Sue, Jules Janin, Sainte-Beuve, Alphonse Karr. Jules Verne y a publié ses premiers textes (dès 1851). 4 Jean-Louis Mongin : Jules Verne et le Musée des familles. Amiens : Centre Rocambole/Encrage Édition 2013, p. 11. Rappelons qu'il s'agit là non de tirages, mais d'abonnés ; un exemplaire se prête, et compte plus d'un lecteur, surtout dans les cabinets de lecture ; la lecture n'est d'ailleurs elle-même qu'un indice de notoriété.

5 Jules Janin : Les Magasins anglais, p. 3. 


\section{Boitard et le Musée des Familles}

Pierre Boitard n'est plus très connu quand, en 1875, Samuel-Henry Berthoud dresse ce portrait de son ami disparu en 1861 :

Il y a un quart de siècle environ, mon vieil ami Pierre Boitard, " grand doubteur, » comme dit Montaigne, était le plus impitoyable mystificateur qui se soit complu à tromper les savants, à les induire en erreur, à les mettre en défaut et à leur rire au nez des mauvais tours qu'il leur jouait.

Il commit entre autres un jour, à leur égard, la damnable malice de semer dans diverses parties des environs de Paris des graines de plantes venues souvent d'une autre moitié du globe ; il les laissa récolter ensuite aux botanistes comme appartenant à la flore parisienne, sauf plus tard à les railler sans merci de leur crédulité. ${ }^{6}$

Pierre Boitard, qui a décrit le diable de Tasmanie comme une espèce à part entière, et qui est habituellement considéré comme l'auteur de la première fiction préhistorique, ${ }^{7}$ Paris avant les hommes, est aussi un des auteurs essentiels du Musée des familles. Le naturaliste rejoint le Musée des familles en 1835 et il publie son dernier texte dans les colonnes du journal en 1848. Il reste encore longtemps après sa mort mentionné dans les tables comme contributeur de la revue ; on le retrouve en 1866 dans la composition de l'illustrateur Bertall présentant les différents contributeurs de la revue. ${ }^{8}$ Boitard a subi le même sort que le Musée des familles : quoique reconnu à son époque, et rappelé à notre mémoire par les travaux de Claude Blanckaert, sa collaboration au Musée des familles reste encore dans l'ombre. ${ }^{9}$ Le portrait que tire de lui Berthoud en « doubteur » et « mystificateur » donne une image facétieuse et ambiguë du naturaliste, image qui concorde avec nombre de ses textes ; ses premières contributions au Musée des Familles prennent cependant d'abord la forme, assez attendue, de leçons d'histoire naturelle.

Ces premières leçons d'histoire naturelle se situent dans la lignée des contributeurs qu'il remplace, Charles Nodier ou Adélaïde Montgolfier. ${ }^{10}$ Néanmoins,

6 Samuel-Henry Berthoud : Les Petites Chroniques de la science. Paris : Garnier Frères 1862, réédition de 1875, p. 610 [en ligne]. URL : http://gallica.bnf.fr/ark:/12148/bpt6k2026439 [consulté le 25/01/2019].

7 La notion de " précurseur » ou de " premier » est largement remise en question ; la notion de " préhistoire » est, de plus, postérieure à Pierre Boitard.

8 Bertall : Une visite au Musée des familles. In : Musée des familles 34 (avril 1867), p. 209 [en ligne]. URL : http://gallica.bnf.fr/ark:/12148/bpt6k5401272f/f234.item.r=boitard [consulté le 25/ 01/2019].

9 Cette présentation reprend en partie une notice que j’ai consacrée au Musée des familles, voir Gisèle Séginger (éd.) : Dictionnaire Flaubert. Paris : Honoré Champion 2017, p. 999-1001. 10 Bory de Saint-Vincent compte également parmi les contributeurs du Musée. 
quand cette dernière pouvait proposer une visite effrayée au Jardin des Plantes pour toute instruction, Boitard met plutôt à profit la fonction érudite de la parenthèse en précisant systématiquement les noms latins des espèces qu'il décrit. Accompagnées de gravures, le plus souvent signées par Theodor Susemilh, ses leçons suivent un déroulement traditionnel : l'animal est présenté au sein de la classification buffonienne, son anatomie est décrite et sa localisation géographique précisée. L'espèce à laquelle il appartient est éventuellement distinguée des espèces qui lui sont proches - les espèces favorites du Musée, crocodiles, chimpanzés, condors ou félins sauvages, sont généralement éloignées du quotidien de ses lecteurs, L'éthologie animale, surtout quand elle est pittoresque, est rappelée ; ce sont surtout l'alimentation de l'animal, ses traits d'humanité - sociabilité comme vie familiale - qui sont susceptibles de retenir le lectorat, ainsi que les meilleures préparations culinaires, stratégies de vénerie ou de défense en milieu sauvage. Boitard n'oublie pas d'utiliser le ressort de l'exotisme, appuyé par des gravures spectaculaires. Celles-ci occupent une demi-page et elles sont même régulièrement en pleine page. Les leçons se veulent scientifiques : Buffon et $\mathrm{Cu}$ vier sont régulièrement mentionnés et les notes de bas de page, comme la précision du nom latin des espèces, appuient la savante fiabilité de la leçon.

Les positions de Boitard se devinent peu dans ces leçons, et elles sont d'ailleurs difficiles à résumer avec précision. Régulièrement rattaché au transformisme et au lamarckisme, Boitard ne mentionne pas dans le Musée des familles l'auteur de la Philosophie zoologique : ses théories naturalistes relèvent pour Claude Blanckaert de l'uniformitarisme et du gradualisme. ${ }^{11}$ Cas particulier, la leçon consacrée au «Chimpansé » [sic] renforce la proximité de l'homme et du singe et postule que l'appellation d'homme des bois désignerait le chimpanzé. La gravure qui accompagne le texte est présentée comme la première publiée en France " avec exactitude »; elle est dessinée et gravée par Susemilh d'après le naturaliste anglais Tyson. ${ }^{12}$ Elle appuie nettement la proximité affichée dans le texte entre chimpanzés et humains : la femelle est debout, elle adopte une posture rattachée à la féminité humaine, avec une légère flexion du bassin, et les jambes coquettement serrées. ${ }^{13}$

Les leçons d'histoire naturelle de Boitard prennent un tournant quasi merveilleux, avec ses Réalités fantastiques : ce sont alors les faits et les espèces les

11 Claude Blanckaert : Devilish Words: Pierre Boitard, "maître Georges" and the Advance of Nature. In : Niklas Bender/Gisèle Séginger (éds.) : Biological Time, Historical Time. Transfers and Transformations in $19^{\text {th }}$ Century Literature. Leyde/Boston : Brill/Rodopi 2019, p. 45-60, p. 54.

12 Pierre Boitard : Le Chimpansé. In : Musée des Familles 2 (1834-1835), p. 127.

13 L'exactitude du dessin est donc toute relative. 
plus curieux qui attirent son attention. Le polype y est, entre autres, présenté avec verve et il est l'occasion d'une réflexion sur la division des espèces et la définition de la vie :

Pour qu'il y ait animalité, il faut qu'il y ait égotisme et unité de vie ; il y n'a égotisme que lorsqu'il y a un centre commun de sensations, ainsi que l'expérience le démontre, car aucun animal pourvu de nerfs ne reprend de bouture ; toute partie qui en est séparée meurt sans retour. Ainsi je dirais : Un animal est un être vivant pourvu de la faculté locomotive, ayant un centre commun de sensation et n'ayant qu'une vie unique ; je donnerais à la classe qui nous occupe un nom quelconque ; celui de polybion, sic] (plusieurs vies), par exemple, ou tout autre qui vous plaira : et je dirais : Un polybion est un être vivant, souvent pourvu de la faculté locomotive, manquant de centre commun de sensation, ayant une vie multiple, et l'azote pour base de sa composition chimique. Ce dernier caractère les trancherait net des végétaux, dont la base chimique est le carbone. ${ }^{14}$

L'ornithorynque, offre, lui, la possibilité de vitupérer contre les classifications scientifiques :

Mais voilà où commence pour eux [les naturalistes] le fantastique, non pas le fantastique de la nature, mais le fantastique de la science. Ce maudit ornithorinque [sic], hélas ! est un mammifère qui n'a point de mamelles, est un quadrupède vivipare qui fait des œufs ! Et puis travaillez donc quarante ans de votre vie à étudier les sciences pour faire un système $!^{15}$

Les exclamations animent une énumération d'animaux surprenants. C'est alors la difficile classification de l'ornithorynque qui légitime sa présence dans la leçon d'histoire naturelle : la science telle qu'elle se construit alors bute devant cet animal hors-normes. Cette intrusion d'auteur montre un glissement : pour un court moment, Boitard sort du registre scientifique ; c'est parce qu'il a déjà adopté la position comique d'un narrateur ridicule qu'il lui est possible de contester la science des nomenclatures.

\section{Excentrer pour vulgariser autrement: Paris avant les hommes et les Études astronomiques}

Boitard vitupère contre une certaine science, qu'il considère comme sèche et aride. Il ne se contente pas de réfuter les classifications : il se met lui-même en

14 Pierre Boitard : Réalités fantastiques - Troisième article. In : Musée des familles 4 (1836-1837), p. 187.

15 Ibid., p. 182. 
scène dans ses textes, multipliant les adresses à ses lecteurs pour contrer l'austérité des nomenclatures par un discours résolument comique, placé au cœur de ses leçons d'histoire naturelle. Ses commentaires, qui miment l'oralité de l'enseignant dans son cours, renforcent le ridicule constant du narrateur dans ses textes. Ces intrusions d'auteur restent cependant une étape intermédiaire, rapidement concurrencée par des leçons d'histoire naturelle à l'ancrage fictionnel bien plus marqué. Ainsi, le conte botanique « Fatima » (1837), qui permet la mise en récit des différentes plantes du désert, remplace les récits de chasse ; Boitard peut prêter la parole au caniche Médor, dans une prosopopée de $1846,{ }^{16}$ pour plaider la cause des chiens et endiguer des massacres, comme ceux de la rue Guénégaud en 1825. C'est surtout par la mise en scène d'un narrateur naturaliste ridicule que Boitard prolonge ces vitupérations, notamment dans deux études spectaculaires, « Paris avant les hommes » (1836) et « Les Études astronomiques » (1839). Le décentrement offert par la fiction est mis à profit par Boitard qui se plaît à subvertir les théories scientifiques alors admises, comme le cuviérisme.

Ces deux récits profitent des ressources offertes par la fiction : " Paris avant les hommes ", première mouture de $1836 \mathrm{du}$ roman posthume de Boitard, ${ }^{17}$ offre un déplacement temporel, aux origines du globe et des espèces, quand « Les Études astronomiques » offrent un voyage spatial, aux confins du système solaire. Ces déplacements, qui confinent au merveilleux, ${ }^{18}$ sont, dans les deux cas, rendus possibles par l'assoupissement du naturaliste et par l'apparition du personnage du diable boiteux, issu du roman de Lesage, Asmodée. ${ }^{19}$ Celui-ci ne soulève plus alors les toits, mais il entraîne le narrateur dans des voyages spatiaux ou temporels ; « Paris avant les hommes » montre l'évolution de la terre, l'apparition des animaux et offre une rencontre rapide avec un homme-fossile. ${ }^{20}$ La forme même du texte montre la volonté de Boitard de fournir une vulgarisation plaisante : les notes de bas de page et la fin du texte explicitent au lecteur les faits scientifiques sur lesquels il s'est appuyé et ce qui ressort de la fiction, tout en permettant

16 L'éditeur du mémoire de Médor (Pierre Boitard) : Le caniche Médor à Messieurs les honorables souscripteurs du Musée des familles. In : Musée des familles 14 (1845-1846), p. 345-349.

17 Boitard est décédé en 1859, son roman a été publié en 1861, voir Pierre Boitard : L’Univers avant les hommes. Paris : Claye 1861.

18 Le merveilleux scientifique ne commencera à être véritablement théorisé qu'avec Maurice Renard au xxe siècle.

19 Le choix du diable boiteux peut avoir été renforcé par la publication d'un almanach, en Suisse puis en France, Le Messager boiteux, à partir de 1708 (quand le roman de Lesage date de 1707).

20 Qui sera développée dans la version de 1861. Le nom d'« homme-fossile » n'apparaît que dans la version romanesque de Paris avant les hommes et dans les «Études astronomiques »; Boitard fait alors allusion à un exposé paru en 1838 dans le Magasin universel. 
une lecture divertissante. Comme toutes les leçons d'histoire naturelle, ces deux textes sont accompagnés de grandes planches.

Boitard s'affiche régulièrement et explicitement comme cuviériste : il ne se détacherait de cet illustre modèle que par un désaccord sur le dessin d'une oreille, désaccord révélé par une note infrapaginale. Cette prudence pusillanime est un leurre : si le nom de Cuvier constitue un patronage officiel et prudent, les tableaux, présentés par le diable, d'une nature en perpétuelle métamorphose le contredisent radicalement. « Paris avant les hommes » présente en effet l'hypothèse, audacieuse, d'une modification des espèces, qui se distinguent progressivement. Alors que le texte se découpe en six tableaux, pour les six périodes terrestres inspirées $\mathrm{du}$ récit de la Genèse, ${ }^{21}$ le passage d'une période à une autre ne peut se faire, d'après le diable boiteux, par une succession de catastrophes :

Rien : autre chose que ce que tu vois ici. La création est lente, elle marche pas à pas ; mais elle est uniforme par toute la terre, parce qu'elle est soumise à une règle uniforme et invariable, sans laquelle elle est possible. Cette règle consiste à procéder du simple au composé, d'abord double, puis triple, puis quadruple, ainsi de suite jusqu'à l'organisation la plus compliquée. ${ }^{22}$

L'uniformitarisme esquissé par la « règle uniforme et invariable », sans être explicitement diachronique, s'oppose au catastrophisme, quand l'évolution supposée « lente » s'oppose aux « révolutions du globe » de Cuvier ; Pierre Boitard se détache ainsi, dans une revue populaire, des théories admises et reconnues. Le refus de la théorie des catastrophes est alors explicite :

Ce n'est que lentement que la mer a successivement abandonné ses vastes plages, ses profonds abîmes, pour couvrir et découvrir des continents. Sans cela la nature entière serait horriblement bouleversée et tous les êtres organisés auraient péri dans ces épouvantables catastrophes. Ne vois-tu pas au contraire que la création des animaux suit une marche régulière et successive, analytique, si je puis me servir de cette expression ; qu'elle commence par les plus simples pour passer aux composés, puis de ceux-ci à de plus composés encore, et qu'enfin elle finira par le plus parfait, l'homme. ${ }^{23}$

Ce refus du catastrophisme géologique implique alors une évolution des êtres vivants en fonction de leurs milieux :

Ne savez-vous pas que les êtres sont modifiés en raison des climats et des milieux qu'ils habitent ? Qui vous a dit que les palmiers de ce temps-là, habitant l'endroit où nous

21 Cette concordance est révélée par le narrateur naturaliste ; Boitard fait alors malicieusement défaillir le diable boiteux.

22 Pierre Boitard : Paris avant les hommes. In : Musée des familles 3 (1835-1836), p. 259.

23 Ibid., p. 266. 
sommes, n'étaient pas organisés de manière à supporter sans inconvénient un froid de 12, de 20 degrés Réaumur ? Qui vous oblige de croire que la terre a fait une cabriole sur son axe parce qu'elle aurait reçu en passant un coup de queue d'une comète ? de faire du globe une boulette refroidie, de l'atmosphère un bain de vapeur, de la mer un consommé servi chaud, et autres billevesées de la même force ? $^{24}$

Là aussi, des pointes contre la science et ses démarches surgissent : les « billevesées », qui résument les positions cuviéristes, sont ridiculisées par leur trivialité croissante. Texte que l'on rattacherait aujourd'hui au transformiste, accompagné par les gravures de Susemilh, " Paris avant les hommes » ouvre donc la voie à l'hypothèse audacieuse d'une métamorphose (on n'ose dire évolution) graduelle des espèces ${ }^{25}$ qui, partant d'êtres hybrides, montre le déploiement des espèces à venir. Pierre Boitard ouvre une brèche dans le cuviérisme institutionnel, en présentant notamment une organisation, d'inspiration lamarckienne, qui va du simple au complexe :

Vois, me dit-il, la nature, comme si elle essayait ses forces, a commencé l'organisation de la matière par les êtres les plus simples. Aucun oiseau ne vole encore dans les êtres les plus simples ; aucun mammifère n'a fait encore retentir les échos de ses cris d'amour ou de colère ; pas un reptile, pas un animal vertébré n’a encore foulé ce désert. Il n'existe pas un être qui ait une respiration aérienne, une voix dont les sons vinssent troubler le silence de la création. À travers ces ondes transparentes tu verras quelques zoophytes ou animaux-plantes, la plupart attachés pour toujours sur la pierre submergée qui les a vu naître. Les uns ressemblent à de longs panaches flottants, les autres à des artistes, à des fleurs dont ils ont les brillantes couleurs et [la] singulière faculté de se reproduire par boutures ; les autres, madrépores, coraux, millépores, ont plusieurs parties entièrement pierreuses, comme pour indiquer qu'ils tiennent encore de la nature des minéraux qui les ont précédés. ${ }^{26}$

Cette organisation du simple au complexe est alors repensée par Boitard comme une marche analytique, marquant le passage de la synthèse à l'analyse : c'est ainsi que les êtres originaires peuvent être des monstres, rassemblant dans leur être les différentes espèces à venir :

En effet, je me vis tout à coup entouré d'êtres fantastiques qui n'ont plus d'analogues vivants.

Regarde celui-ci, me dit le génie ; il fait le passage naturel des poissons aux reptiles ; il est encore moitié brochet, moitié tortue, et il tiendrait plus de cette dernière que du premier s'il ne respirait par des branchies au lieu de poumons. C'est un megalichtys, et,

24 Ibid., p. 260.

25 Il ne parle pas lui-même d'évolution ou de transformation.

26 Ibid., p. 259. 
comme tu peux le voir à travers la transparence des ondes, il a encore conservé de son ancienne nature de reptile la pesanteur dans la natation et la lenteur des mouvements. ${ }^{27}$

Les êtres montrés par le diable boiteux sont les êtres intermédiaires, hybrides, qui marquent les temps de la synthèse originaire. À mi-chemin entre les espèces, le megalichtys est ainsi la démonstration du transformisme naturaliste.

" Paris avant les hommes » montre par ailleurs le ridicule du naturaliste lui-même, tant par sa volonté de classer le dinothérium que par ses fréquents trébuchements. Celui qui aurait pu être le guide de la transmission des savoirs n'est en effet pas le naturaliste officiel, qui se trouve privé de toute autorité scientifique valide, mais un personnage intertextuel et diabolique. Il apparaît néanmoins qu'Asmodée est un porte-parole commode : le diable a bien le champ libre pour contester les théories les plus admises : (enchaînement de ces signes de ponctuation impossible)

Mon pauvre ami, me dit le démon, c'est positivement parce que vous ne pouvez juger, dans la faiblesse de votre intelligence humaine que par analogie, c'est positivement pour cela, dis-je, que votre prétendue science n'est qu'un pur ramassis de pauvretés au milieu desquelles se trouvent enfouies trois ou quatre vérités, ainsi que des diamants perdus dans la fange. Quand tu connaîtras le plus petit brin de matière, quand tu auras reconnu toutes les lois qui lui donnent ses propriétés, quand tu te comprendras toi-même, alors tu sauras ; tu n'auras pas besoin de tes analogies qui t'égarent plus souvent qu'elles ne t'éclairent. Tu sauras que le dinothérium n'était ni un éléphant, ni un morse, ni une baleine, ni une taupe, mais un dinothérium. Tu comprendras qu'ailleurs que dans les bois de Meudon les lièvres peuvent aller à la chasse aux chiens, car tu sauras que la matière peut s'organiser de mille millions de milliards de manières avec des analogies tout à fait différentes de celles que tu connais, et alors... ${ }^{28}$

Quand « Paris avant les hommes », histoire d'avant l'histoire, restituait un passé lointain, « Les Études astronomiques » imaginent un univers inaccessible. La forme reste cependant classique - ce sont les savoirs qui sont inventés - et un passage sur Vénus permet de voir derrière les Vénusiens « certaine figure de l'homme fossile» :

Les Vénusiens étaient des animaux bipèdes comme les Cafres et les Pongos : mais ils différaient des uns et des autres par des caractères assez tranchés. Leur taille ne dépassait pas cinq pieds six ou sept pouces, et leur corps, d'un brun rougeâtre, était entièrement couvert d'un poil fauve tirant sur le blond. Leur museau était plus proéminent que celui des Cafres, mais moins que celui des Pongos ; ils manquaient de cuisses et de mollets, comme les habitans de quelques îles de l'Australasie ; leurs pieds étaient fort longs, ainsi que les pieds des insulaires de plusieurs îles de la Nouvelle-Zélande, et, chose fort

27 Pierre Boitard : Paris avant les hommes. In : Musée des familles 5 (1837-1838), p. 52.

28 Pierre Boitard : Études astronomiques. In : Musée des familles 7 (1839-1840), p. 40. 
remarquable, leur pouce du pied était opposable aux autres doigts, ainsi que chez quelques sauvages de l'Amérique méridionale. Enfin je ne saurais vous en donner une idée plus juste que certaine figure de l'homme fossile, que j'ai donné quelque part. Une peau d'ours pendait sur leurs épaules, et ils avaient à la main des bâtons, ou plutôt des massues qui paraissaient avoir été assez proprement taillés avec des pierres tranchantes. ${ }^{29}$

La présentation des Vénusiens montre que les "Études astronomiques » ne sont pas une simple divagation : les espaces lointains, comme les tableaux du passé, permettent d'illustrer des théories naturalistes hétérodoxes. L'« hommefossile " n'est plus ici une hypothèse : il devient au contraire le référent pour un extraordinaire plus lointain. Les «Études astronomiques » se placent ainsi sous le signe de la conjecture scientifique à partir d'un postulat merveilleux. Comme " Paris avant les hommes ", le diable est le subterfuge fictionnel permettant d'établir un imaginaire scientifique subversif : derrière le boiteux se cache le Boitard.

\section{La vulgarisation scientifique excentrique : contester la science}

Quasi hapax dans la production de Pierre Boitard pour le Musée des familles, " Paris avant les hommes » et les "Études astronomiques " sont des fictions sortant des formats de la vulgarisation scientifique - tout en délivrant des savoirs dans un registre conversationnel assez habituel, adopté par la plupart des leçons d'histoire naturelle livrées au Musée. Nombre de ses leçons ne s'inscrivent en effet pas pleinement dans ce genre didactique. S'ils finissent toujours par vulgariser, certains textes montrent en effet une certaine réticence à délivrer la leçon initialement prévue et détournent comiquement le propos annoncé. Les sorties virulentes des Réalités fantastiques se trouvent ainsi prolongées dans Les Insectes musiciens. Dès la gravure inaugurale, le ton est donné : cette leçon d'histoire naturelle se fera plaisante. Elle débute en effet par une longue digression faussement autobiographique :

C'est une bien belle chose que la science ! je m'en suis occupé toute ma vie, et si je suis resté un ignorant, comme je le pense et comme le disent mes bons amis, c'est bien un peu ma faute : voici comment. Il y avait autrefois dans mon village un maître d'école trèssavant, nommé M. Gobemouche. Il savait peu de français, moins de latin, point de grec ni de mathématiques, et il ne soupçonnait pas une philosophie, cela est vrai ; mais en

29 Ibid., p. 38-40. 
récompense il nommait à première vue et sans la moindre hésitation, avec toute leur synonymie, la mouche qui lui piquait le mollet, le cousin qui bourdonnait autour de son bonnet de nuit, et le taon qui relançait sa vache dans son verger à travers ses choux. Pendant la classe, il dissertait à perte de vue et des heures entières sur le thorax, le prothorax, le labre et le pygidium. ${ }^{30}$

Envoyé à Paris par son père pour devenir naturaliste, " ainsi qu'il est d'usage chez les parents ", ${ }^{31}$ le jeune homme se décourage devant les 250000 noms d'espèces à apprendre :

Moi qui avais eu tant de peine à me fourrer dans la tête la Monographie illustrée du lapin de garenne, publiée par mon père, je tombai de mon haut et restai pendant plusieurs jours dans la stupeur et le découragement. Enfin, j’appris de M. Piquinsecte, professeur de hannetonologie à l'Athénée, qu'il y a deux ordres de naturalistes, savoir : $1^{\circ}$ le savant par état, collectionneur, étiqueteur, descripteur, classificateur, nomenclateur et souvent professeur. Celui-ci possède la science infuse de par les règlements ministériels et la loi, bon gré, mal gré, et il en vit confortablement. $2^{\circ}$ Le flâneur scientifique, pauvre hère se bornant à l'observation et à l'analyse des faits, à leur généralisation et à leur génération philosophique ; à leur application aux choses de ce monde, telles que la décortication des pois, la teinture de cochenille et le vésicatoire de cantharides, toutes choses qui ne sont pas du ressort du savant, car le vrai savant ne doit pas descendre assez bas pour pouvoir être saisi par l'utilité. ${ }^{32}$

De ces deux modèles de savants, tous deux moqués, c'est le second - le « flâneur scientifique, pauvre hère se bornant à l'observation et à l'analyse des faits » - qui a la faveur de Boitard. La promenade, souvent contemplative, se confronte alors à la classification, à la nomenclature et à l'exigence d'utilité. Les promenades, qui trouvaient leur modèle dans les Rêveries $d u$ promeneur solitaire (1782) de Rousseau, se font véritablement scientifiques en valorisant un vagabondage méthodique, à la marge des expériences des naturalistes et des nomenclateurs :

J'avais déjà beaucoup flâné dans les forêts qui entourent mon village, je me mis à flâner dans les rues de Paris, faisant des observations tantôt sur le crocodile et le boa de la foire, tantôt sur l'éléphant et le lion de la ménagerie, quelquefois, par pure distraction, sur polichinelle, l'âne savant et les caricatures de Martinet. Pendant mes longues années de flânerie, j'ai amassé çà et là, sans trop savoir où, quelques bribes scientifiques dont je vous ai gratifié ; mais je ne pense pas que je vous aie déjà parlé de moi, et, aujourd'hui, je me sentais ce besoin irrésistible, inhérent au métier d'écrivain. Comme il est à peu près certain que je ne publierai jamais ni mes mémoires ni mes émotions (je suis très-peu nerveux), ni mes voyages en Sicile ou en Orient, vu que je ne me suis jamais éloigné de

30 Pierre Boitard : Les Insectes musiciens. In : Musée des familles 12 (1844-1845), p. 17. 31 Ibid.

32 Ibid., p. 18. 
quinze cents pas des barrières de Paris, j'ai saisi l'occasion aux cheveux, et voilà. Maintenant, parlons musique. ${ }^{33}$

$\mathrm{Au}$ vagabondage champêtre succède la promenade urbaine : l'objet d'étude perd ainsi, de l'éléphant et du lion « de la ménagerie » aux « caricatures de Martinet », tout sérieux scientifique, dans une nature déjà contextualisée. L'éthos du scientifique s'estompe devant le travail de l'écrivain polygraphe. La mise en scène du récit, interrompu par ce long discours biographique, accompagne alors une critique scientifique mêlée d'autodérision. La suite du texte parle effectivement de musique, mais aussi - enfin - d'entomologie : elle se construit comme un commentaire explicatif de l'image grotesque qui ouvrait la leçon et qui représentait un orchestre d'insectes. Les différents insectes sont présentés et caractérisés : l'image surprenante s'explique ainsi par l'anatomie des animaux, proche des différents instruments artificiels. L'introduction comique permet alors de justifier un rapprochement plaisant et didactique, tout en donnant un ton oral et badin à la leçon naturaliste.

Cette entrée en matière ne semble cependant pas seulement plaisante : elle permet également de valoriser des savoirs traditionnels - comme ceux des apothicaires - en prenant en charge un discours scientifique. Les sciences naturelles académiques entérinent en effet la classification systématique, qui s'impose alors à la communauté scientifique. La flânerie ne se limite donc pas à un simple agrément de la vulgarisation scientifique : elle réalise la préférence pour une science moins académique, plus littéraire et contemplative. Le dénigrement du « vrai savant » comme de la figure du professeur, possesseur de la «science infuse par les règlements ministériels et la loi », affirment l'écart entre la science et son objet - la nature. Ce discours méta- (et anti-) scientifique favorise ainsi une autre science, non académique, et il se manifeste par sa réticence même à livrer la leçon naturelle attendue. La vulgarisation scientifique se rapproche ainsi du modèle du récit excentrique étudié par Daniel Sangsue : l'auteur se met en scène, se désigne comme tel et s'adresse à son lecteur pour empêcher la vulgarisation scientifique. Sous-genre influencé par le récit excentrique de la première moitié $\mathrm{du} \mathrm{XIX}{ }^{\mathrm{e}}$ siècle, cette vulgarisation scientifique excentrique se caractérise par la " discontinuité » (la leçon est interrompue pour une adresse au lecteur), les « digressions » et l' " hypertrophie du discours narratorial $»{ }^{34}$ Contrairement au récit excentrique, qui

33 Ibid.

34 «Dans les pages qui suivent, et particulièrement dans la seconde partie de ce travail, on se propose d'analyser des récits qui ont été écrits entre 1800 et 1850 et qui se caractérisent par leur discontinuité, une composition problématique, des digressions, une hypertrophie du discours narratorial et une atrophie de l'histoire racontée, une mise en question des personnages, 
conteste le « romanesque " et suspend l'immersion supposée de la fiction, ${ }^{35}$ la vulgarisation que l'on pourrait appeler excentrique ne s'oppose pas au récit, ni même aux mérites de la vulgarisation scientifique, ${ }^{36}$ mais à la science ellemême, dans son élaboration comme dans sa transmission (professorale et sèche). La nomenclature et la taxinomie en sont alors les premières cibles.

La vulgarisation scientifique excentrique permettrait alors d'esquisser les traits d'un micro-genre littéraire, surtout productif dans les années du milieu du XIX ${ }^{\mathrm{e}}$ siècle, contestant les transformations paradigmatiques des sciences, notamment naturelles. ${ }^{37}$ Passé inaperçu, ce petit genre serait en effet diffusé par des relais non institutionnels, comme la presse populaire, alors régulièrement irrévérencieuse, et il passerait d'abord pour comique - excentrique - avant d'être perçu comme une vulgarisation scientifique. Ce type de vulgarisation reste cependant un discours permettant une transmission de savoirs : la vulgarisation scientifique excentrique finit toujours par donner la leçon attendue. Les textes d'Alphonse Karr, autre collaborateur du Musée, autre vulgarisateur comique et lui-même éditeur de revues, appartiendraient eux aussi à cette veine, dont Les Souvenirs entomologiques de Jean-Henri Fabre constitueraient un autre avatar, plus lointain.

C’est avec « La très-véridique histoire des dix-neuf infortunes de Jannot le harponneur » que la spécificité de la vulgarisation scientifique de Pierre Boitard se manifeste le plus clairement : l'ensemble du texte, récit du malheureux Jannot, reprend en effet les grandes lignes du roman picaresque, épicées de vulgarisation scientifique. ${ }^{38}$ Le début de ce récit comique se rapproche alors de l'excentricité littéraire, grâce à un jeu énonciatif particulièrement retors. Le récit débute en effet par un dialogue entre le naturaliste, qui regrette de ne pas avoir voyagé, et sa vieille mère aveugle :

- Mon Dieu ! mon Dieu ! ai-je bien entendu ? Quoi ! tu voudrais quitter ton pays, tes amis, ta famille, pour courir après une vaine fumée qui n’a rien de réel ni d'utile?

ainsi que par d'autres dispositifs qui visent globalement à une contestation du romanesque. » (Daniel Sangsue : Le Récit excentrique. Gautier, De Maistre, Nerval, Nodier. Paris : Corti 1987, p. 9.)

35 Sur cette question, voir notamment Françoise Lavocat : Fait et fiction. Paris : Seuil 2016.

36 La question est alors régulièrement posée, et elle trouve des traces notamment dans l'Avenir de la science de Renan, rédigé à la fin des années 1840, mais publié en 1890.

37 Sur l'établissement d'une science normale, voir les analyses de Thomas Kuhn : La Structure des révolutions scientifiques. Traduit par Laure Meyer. Paris : Flammarion 2008.

38 Il s'agit probablement d'un récit inspiré du voyageur et naturaliste Victor Jacquemont. 
- Songe donc, mère, que les moufettes sont si mal connues ! Quelle gloire pour celui qui débrouillerait leur synonymie sur les lieux mêmes où elles vivent !

- Eh ! qu'importe les moufettes ? Je gage que sur trente-quatre millions de Français, il ne se trouverait pas dix personnes qui voulussent se donner la peine de se baisser pour en ramasser une dans le ruisseau de la rue. Tiens, petit (notez que le petit de ma mère a cinq pieds cinq pouces et cinquantesix ans), je trouve que tu deviens doublement bête, comme un classificateur et un nomenclateur, depuis que tu t'es fourré cette malheureuse science dans la tête. Tu étais si gentil autrefois, avec tes jolis cheveux blonds bouclés, tes petites mains blanches et potelées dont tu me caressais la figure, tes petits mots charmants que tu commençais à peine à pouvoir articuler. ${ }^{39}$

Le rappel au bon sens maternel ridiculise alors les prétentions érudites et naturalistes du narrateur : la classification et la nomenclature emplissent la tête plus qu'elles ne la forment. Les désagréments des voyages, traditionnellement rappelés dans les voyages comiques et excentriques, conduisent alors à la sage décision d'un voyage autour du jardin :

J'admirai combien l'amour maternel est ingénieux, car c'était pour la première fois de ma vie que j'entendais la vieille mère parler science. Sans croire à ses sophismes inspirés par l'affection, je pris la ferme résolution de ne voyager, quant à présent, que dans mon jardin, qui a vingt pas de largeur et trente de longueur. Et ne vous imaginez pas que ce voyage n'ait pas ses dangers, ses écueils et ses tempêtes. Plus d'un écrivain, vous le savez aussi bien que moi, a fait naufrage sans même sortir de son cabinet, et s'est noyé à tout jamais dans son encrier. Nonobstant cela, je mets à la voile, je pars, et je prie bien humblement mes lecteurs de ne pas faire chavirer ma frêle nacelle scientifique. ${ }^{40}$

Ce voyage débute sous la houlette du cicérone maternel ; la description d'une araignée se trouve brusquement interrompue par l'arrivée de Jannot qui, lui-même, a voulu étudier « des mammifères d'autant plus intéressants, que leur synonymie est très-embrouillée en Europe [à savoir] les moufettes . $^{41}$ Le début de ce récit se présente donc comme une entrée habituelle d'un sous-genre littéraire mineur, inspiré du Voyage autour de ma chambre de Xavier de Maistre (1794), et il est plus précisément une reprise directe du Voyage autour de mon jardin d'Alphonse Karr

39 Pierre Boitard : La très-véridique histoire des dix-neuf infortunes de Jannot le harponneur. In : Musée des familles 13 (1845-1846), p. 147.

40 Ibid.

41 Ibid., p. 150. 
(1845). ${ }^{42}$ Le refus du voyage, l'annonce d'un voyage autour du jardin, l'araignée décrite sont en fait les éléments d'une parodie d'un texte lui-même déjà excentrique. Cette parodie est claire pour un familier du texte de Karr, ami et collègue de Pierre Boitard. Les allusions au genre littéraire du voyage en miniature sont une caractéristique du genre circumcamériste ; Boitard établit alors un jeu de connivence supplémentaire en évoquant parodiquement une autre parodie. La description de l'araignée, qui était un temps du Voyage autour de mon jardin, se trouve interrompue (et le voyage avec lui) par l'arrivée du malheureux Jannot, ancien camarade du narrateur, qui débute alors le récit de ses lointains et aventureux voyages - les dix-neuf infortunes du titre. La tentative d'un voyage naturaliste dans un jardin se trouve ainsi avortée par un autre récit, qui légitime le récit cadre - les voyages sont dangereux - mais le remplace : le pied-de-nez adressé à l'auteur des Tilleuls et des Guêpes est manifeste.

C'est dans un texte signant le retour du professeur Gobemouche et de son élève-narrateur, avatar de Boitard, que se trouvent les critiques les plus virulentes de la science et des savants. Gobemouche conseille à son disciple de ne pas « disséquer la science, [de] ne pas [s]e perdre dans de stupides détails de nomenclature et de classification, [de] rétrécir [s]on esprit et [de] lui faire perdre la faculté de généraliser »; l'ancien élève, qui se souvient de ces conseils, rappelle alors la maigre réception de ses écrits. Ce constat amer est l'occasion d'une nouvelle digression métascientifique ; elle conduit ensuite le naturaliste à apparemment quitter le registre de la vulgarisation scientifique pour s'orienter bouffonnement vers une carrière politique :

Or, j'ai eu tout comme un autre mes rêves de gloire et d'ambition scientifique. Alors je voulus essayer de mettre dans mes écrits un peu de philosophie, un peu de pensées, un peu de logique, un peu de critique, un peu de grammaire, un peu de style, un peu d'intérêt dramatique, enfin, l'utile dulci, comme dirait un cuistre. Je ne sais trop quel gâchis j'ai fait de tout cela, mais, ce que je sais bien, c'est que ces innovations hardies m'ont perdu. O ciel ! j'en frémis encore quand j'y pense ! Tous les écrivassiers zoologistes, botanistes, géologistes, coureurs de fauteuils, de chaires, de pensions et de rubans, lancèrent à la fois leur foudroyant anathème sur mon pauvre individu ; à l'unanimité des voix je fus déclaré linnéiste, buffoniste, philosophiste, fétichiste, déiste, matérialiste, ignorantiste, rétrogradatiste, etc., etc. Il ne me resta, pour fiche de consolation, que l'approbation du public instruit, celle de quelques savants qui n'ont jamais balayé les greniers ni ratissé les allées du Jardin des Plantes, et celle, je crois, de soixante mille lecteurs du Musée des Familles, mes futurs commettants. Ma position, comme vous le voyez, est devenue

42 À ce sujet, voir Jérémy Chateau : Représentations de l'homme immobile. Inaction et réclusion dans la littérature occidentale des XVIII ${ }^{e}$ et XIX ${ }^{e}$ siècles. Thèse de doctorat en littérature générale et comparée : Université Bordeaux Montaigne 2016. 
intolérable, et, je le répète, voilà pourquoi je me lance dans une nouvelle carrière, celle de la haute politique, pour arriver à la Chambre basse. ${ }^{43}$

Politique bouffonne, qui se fait le prétexte à l'observation des sociétés des insectes et des animaux, comparées aux différents régimes politiques - la vulgarisation scientifique finit toujours par livrer les savoirs attendus. Là encore, la vulgarisation se fait attendre, et la prétérition, qui retarde le discours scientifique, permet alors de contester la science et la politique institutionnelle des « nomenclateurs ». Avant la politique, le principal refuge du narrateur est, explicitement, le Musée des familles : c'est dans les marges des publications populaires que se trouvent les derniers soubresauts contre la généralisation de la classification des sciences naturelles.

Le genre de la vulgarisation excentrique rassemble ainsi des textes scientifiques qui refusent ou se jouent de leur scientificité. Il s'agit alors d'interrompre la transmission des savoirs, non " pour parler de son chien, de ses pantoufles ou de sa maîtresse », comme le penseront les deux compères de Flaubert penchés sur les romans humoristiques, ${ }^{44}$ mais de questionner, de critiquer et de moquer la science dans sa méthode comme dans ses pratiques. Comme les narrateurs des romans excentriques, Boitard « étal[e] sa personne » et se met en scène. Ce sousgenre qu'est la vulgarisation excentrique trouve alors sa place dans des revues permettant, contre toute attente, des voix contestataires (tant qu'elles restent littéraires), à un moment particulier, celui de l'établissement du cadre d'une " science normale ", tout en étant le fait d'auteurs eux-mêmes relativement marginaux, bien que parfois très lus, comme Alphonse Karr ou Jean-Henri Fabre. La vulgarisation scientifique excentrique reste scientifique : elle ne se refuse pas la science, mais une certaine science, dont le modèle et les méthodes deviennent dominants. La mise en récit littéraire, qui rend possible cette contestation de l'académisme, renouvelle un genre textuel alors en vogue, pour permettre autant la transmission de savoirs qu'une réflexion sur leur portée et leur pratique.

Fictions savantes, satires scientifiques, vulgarisations épistémologiques, les textes de Boitard ne peuvent se réduire à une forme fixe ; cadres hors-normes pour des théories subversives, les leçons et études d'histoire naturelle de Boitard pour le Musée des familles apportent à un nombre conséquent de lecteurs une réflexion épistémologique plaisante, et pourtant à prendre au sérieux. La vulgarisation scientifique excentrique de Boitard, comme ses fictions, déroutent les attentes

43 Pierre Boitard : De la démocratie, de la monarchie et du gouvernement constitutionnel. In : Musée des familles 14 (1845-1846), p. 11.

44 Gustave Flaubert : Bouvard et Pécuchet. Édité par Claudine Gothot-Mersch. Paris : Gallimard 1979, p. 204-205. 
encore mal établies de la littérature scientifique populaire. Les textes de Boitard révèlent ainsi non seulement son rôle de passeur, mais un moment particulier de l'histoire des sciences naturelles. La marginalité de Boitard se traduit par une excentricité littéraire et scientifique qui semble s'éteindre dans la seconde moitié du $\mathrm{XIX}^{\mathrm{e}}$ siècle. S'il a le goût du merveilleux, Boitard, « mystificateur » comme le disait Berthoud, préfère aux savoirs la démarche scientifique du doute, à la gravité des nomenclatures et à la fixité des espèces les bizarreries des hybrides et une science rieuse.

\section{Bibliographie}

Bertall : Une visite au Musée des familles. In : Musée des familles 34 (avril 1867), p. 209. Berthoud, Samuel-Henry : Les Petites Chroniques de la science. Paris : Garnier Frères 1862, réédition de 1875 [en ligne]. URL : http://gallica.bnf.fr/ark:/12148/bpt6k2026439 [consulté le 25/01/2019].

Blanckaert, Claude : Devilish Words: Pierre Boitard, "maître Georges" and the Advance of Nature. In : Niklas Bender/Gisèle Séginger (éds.) : Biological Time, Historical Time. Transfers and Transformations in $19^{\text {th }}$ Century Literature. Leyde/Boston : Brill/Rodopi 2019, p. 45-60.

Boitard, Pierre : Le Chimpansé. In : Musée des Familles 2 (1834-1835), p. 126-127.

Boitard, Pierre : Paris avant les hommes. In : Musée des familles 3 (1835-1836), p. 257-281.

Boitard, Pierre : Réalités fantastiques - Troisième article. In : Musée des familles 4 (1836-1837), p. 326-342.

Boitard, Pierre : Paris avant les hommes - Second article. In : Musée des familles 5 (1837-1838), p. 45-63.

Boitard, Pierre : Études astronomiques - Voyage dans le soleil. In : Musée des familles 6 (1838-1839), p. 65-80.

Boitard, Pierre : Études astronomiques. In : Musée des familles 7 (1839-1840), p. 129-143.

Boitard, Pierre : Les Insectes musiciens. In : Musée des familles 12 (1844-1845), p. 17-23.

Boitard, Pierre : La très-véridique histoire des dix-neuf infortunes de Jannot le harponneur. In : Musée des familles 13 (1845-1846), p. 147-157 et 169-177.

Boitard, Pierre : De la démocratie, de la monarchie et du gouvernement constitutionnel. In : Musée des familles 14 (1845-1846), p. 9-16 et 33-40.

Boitard, Pierre : L'Univers avant les hommes. Paris : Claye 1861.

Bosson, Yves/Abdelouahab, Farid : Dictionnaire visuel des mondes extra-terrestres. Paris : Flammarion 2010.

Chateau, Jérémy : Représentations de l'homme immobile. Inaction et réclusion dans la littérature occidentale des $X V I I I^{e}$ et $X I x^{e}$ siècles. Thèse de doctorat en littérature générale et comparée : Université Bordeaux Montaigne 2016.

Fayolle, Azélie : Musée des familles. In : Gisèle Séginger (éd.) : Dictionnaire Flaubert. Paris : Honoré Champion 2017, p. 999-1001.

Flaubert, Gustave : Bouvard et Pécuchet. Édité par Claudine Gothot-Mersch. Paris : Gallimard 1979. 
Janin, Jules : Les Magasins anglais. In : Musée des familles 1 (1833-1834), p. 3-6.

Kuhn, Thomas : La Structure des révolutions scientifiques. Traduit par Laure Meyer. Paris :

Flammarion 2008.

Lavocat, Françoise : Fait et fiction. Paris : Seuil 2016.

Médor [= Pierre Boitard] : Le caniche Médor à Messieurs les honorables souscripteurs du Musée des familles. In : Musée des familles 14 (1845-1846), p. 345-349.

Mongin, Jean-Louis : Jules Verne et le Musée des familles. Amiens : Centre Rocambole/Encrage Édition 2013.

Raichvarg, Daniel/Jacques, Jean : Savants et ignorants. Une histoire de la vulgarisation des sciences. Paris : Seuil 1991.

Sangsue, Daniel : Le Récit excentrique. Gautier, De Maistre, Nerval, Nodier. Paris : Corti 1987. 